\title{
An Experimental Study of Triangular Airfoils for Mars Airplane
}

\author{
By Hidenori HIDAKA ${ }^{1)}$ and Masato ОKАмото ${ }^{2)}$ \\ ${ }^{1)}$ Graduate School, Kanazawa Institute of Technology, Nonoichi, Japan \\ ${ }^{2)}$ Kanazawa Institute of Technology, Nonoichi, Japan
}

(Received June 24th, 2013)

\begin{abstract}
The objective of this study is to clarify the aerodynamic characteristics of the triangular shaped airfoil at very low Reynolds number corresponding to the Mars airplane flying in very low atmospheric density on Mars. Two-dimensional aerodynamic forces and pitching moments of the thin angular airfoils and the triangular airfoils with various thicknesses were studied through wind tunnel tests conducted in very low Reynolds number range of 3,000 - 20,000. Although the maximum lift coefficient and lift-to-drag ratio were large even for the triangular airfoil composed of a line pattern at the present Reynolds number, these aerodynamic components were affected in the airfoil thicknesses by changing the Reynolds number. The non-linear lift and pitching moment curves were characterized in low Reynolds number flows. It was found that the non-linearity of the aerodynamic curves was caused by the existence of the leading-edge separation bubble by measuring the pressure distributions of the airfoil at various Reynolds numbers.
\end{abstract}

Key Words: Airfoil, Low Reynolds Number, Wind Tunnel Test

\section{Nomenclature}

$\begin{array}{cll}C_{d} & : & \text { drag coefficient } \\ C_{f} & : & \text { friction drag coefficient } \\ C_{l} & : & \text { lift coefficient } \\ C_{m, 0.25 c} & : & \text { pitching moment coefficient at } 25 \% \text { chord } \\ C_{p} & : & \text { pressure coefficient } \\ c & : & \text { chord length } \\ d & : & \text { drag of two-dimensional wing } \\ h & : & \text { height of the airfoil } \\ l & : & \text { lift of two-dimensional wing } \\ m & : & \text { pitching moment of two-dimensional wing } \\ R e & : & \text { Reynolds number } \\ S & : & \text { wing area } \\ t & : & \text { thickness of the airfoil } \\ U & : & \text { wind velocity } \\ \alpha & : & \text { angle of attack } \\ \rho & : & \text { atmospheric density }\end{array}$

\section{Subscripts}

$\max :$ maximum value

min : minimum value

\section{Introduction}

The Reynolds number based on the chord length of the wings of the airplane for Mars exploration planned by JAXA is approximately 20,000 and the Reynolds number for the chord of the propeller blade is 3,000 , because the atmospheric density on Mars is nearly $1 \%$ that of Earth ${ }^{1)}$. When the Reynolds number of the wing is 10,000 or less, the aerodynamic characteristics of the wing will correspond to those of insect wings. There are a few experimental studies on the aerodynamic characteristics of wings at Reynolds numbers less than 10,000. The two-dimensional aerodynamic characteristics of a wing at Reynolds numbers $(R e) R e \leq$ 10,000 have been investigated in a wind tunnel by Okamoto et al. ${ }^{2}$, Azuma et al. ${ }^{3)}$ and Kesel ${ }^{4)}$, and in a water tank by Sunada et al. ${ }^{5)}$, and Okamoto et al. ${ }^{6}$ for three dimensional wings.

Thick streamlined airfoils such as Clark-Y $12 \%$ with leading-edge radii are intended for use at high Reynolds numbers, exhibit poor performance at low Reynolds numbers due to the laminar separation in flows. However, the performance of the streamlined airfoil is improved by decreasing the airfoil thickness. For example, Clark-Y 6\% shows a comparatively high lift-to-drag ratio even at low Reynolds numbers ${ }^{2}$. Such streamlined airfoils with low thickness ratios have been used for small model airplanes by experienced enthusiast modelers. On the other hand, the wing sections of insects such as dragonflies are thin and a corrugated profile. These distinctive profiles are known to perform well at low Reynolds numbers ${ }^{3)}$. It is considered that the airfoil does not need to be streamlined at Reynolds numbers less than 10,000. Therefore, the thin angular airfoils made by bending a thin flat plate and the triangular airfoils having a flat base were selected for the present study. The later will be effective for maintaining the strength of the wing of a manmade aircraft such as Mars airplane. The aerodynamic characteristics of a thin airfoil and a thick airfoil having an upper surface of same shape were compared in this study.

It is well known that the lift slopes $a=\partial C_{l} / \partial \alpha$ of airfoils remain constant at changes of the angle of attack that are less than the stall angle at high Reynolds numbers, whereas the lift slopes are not constant at low Reynolds numbers. The nonlinearity of the lift curve is caused by the leading-edge vortex at the sharp leading-edge. For example, Crompton and Barett ${ }^{7)}$ 
revealed the existence of a leading-edge separation bubble on the flat plate with a sharp leading-edge at $R e=10,000$ 550,000 by measuring the pressure distribution. The separated laminar flow at the sharp leading-edge changes to a turbulent flow and reattaches at the rear point of the surface. The location of the reattachment moves to the trailing-edge as the angle of attack increases. The vortex lift by the leading-edge separation bubble adds to the lift, and a non-linear lift curve is slightly seen even for the flat plate at low Reynolds numbers ${ }^{6}$.

The purpose of the present study is to ascertain the two-dimensional aerodynamic characteristics of angularshaped airfoils at low Reynolds numbers corresponding to the size of an insect by conducting wind tunnel tests. The lift, drag and pitching moment acting on the wing were measured by changing the Reynolds numbers between 3,000 and 20,000. These results were compared with those of a thin flat plate.

\section{Experimental Materials and Methods}

The wind tunnel used for this study is a low-speed, small pusher-type wind tunnel. The test section is $280 \times 280 \mathrm{~mm}$ square with $500 \mathrm{~mm}$ long. The wind velocity $U$ in this section can be varied from 1 to $10 \mathrm{~m} / \mathrm{s}$, and the turbulence intensity is $0.3 \%$ or lower. The balance system comprises a load measuring device and the driving mechanism on which the wing model was mounted, as shown in Fig. 1. The load measuring device was made by authors from some aluminum blocks on which the strain gauges attached, and the lift $l$, the drag $d$ and the pitching moment about the quarter-chord $m_{0.25}$ are measured. For a better delineation of the wind tunnel and measuring device used in this study, please refer to the refs. 2 and 6 , where the principle used in the constitution of the measuring device is similar to that used here. To vary the angle of attack, the wing model is rotated by the driving mechanism of the stepping motor controlled by the programmable logic controller. The output signals of the measuring device are analyzed by a personal computer through a bridge circuit, strain amplifier and 16-bit analog-to digital converter. Aerodynamic data at various $\alpha$ were obtained by averaging 300 datasets measured at a sampling frequency of 1,000 Hz. An averaging time of $3 \mathrm{~s}$ was found to give sufficiently high repeatability of the output data. The

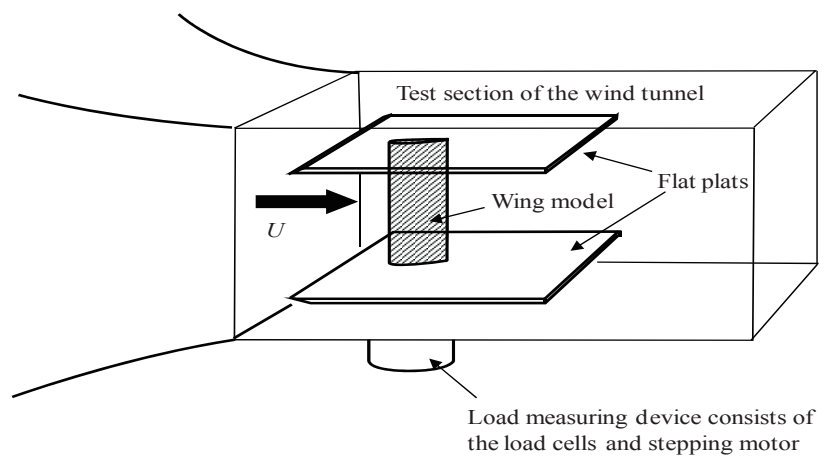

Fig. 1. The balance system. minimum readable values are $3.6 \times 10^{-5} \mathrm{~N}$ for lift and drag, and $7.0 \times 10^{-6} \mathrm{Nm}$ for the pitching moment. It was confirmed that the output signals of load cells had high linearity in the measurement range.

The non-dimensional aerodynamic coefficients $\left(C_{l}, C_{d}\right.$, $C_{m, 0.25 \mathrm{c}}$ ) are given as follows:

$$
C_{l}=\frac{2 l}{\rho U^{2} S}, \quad C_{d}=\frac{2 d}{\rho U^{2} S}, \quad C_{m, 0.25 c}=\frac{2 m_{0.25 c}}{\rho U^{2} S c}
$$

where $\rho$ is the density and $S$ is the wing area.

For obtaining the characteristics of two-dimensional wings, the wing model attached to the measuring device was placed between two flat plates with a sharp leading-edge constructed in the test section as shown in Fig. 1. The clearance between the wing tip and flat plate is below $2 \%$ chord. The effect of this clearance on the measurements was confirmed to be negligible. It was subsequently confirmed using a hot-wire anemometer that the distribution of the flow velocity between two plates is constant except in the range of $4 \mathrm{~mm}$ near the surface of the plate, which is equivalent to $93 \%$ of the wing width of the models. The model wing with several $0.5 \mathrm{~mm}$ diameter holes was used to obtain the pressure distribution of the wing. The pressures were measured using an electric manometer (Okano Works, LTD., DMP200N). The minimum readable pressure was $0.01 \mathrm{~Pa}$ and the response time was $1.0 \mathrm{~s}$. The mean pressures of output data in $5 \mathrm{~s}$ were used in this study.

Figure 2 shows the airfoils used in this study: (a) the flat plate is rectangular with no sharp leading-edge in the section profile, and a thickness $t$ is $1.3 \%$ of the chord $c$, (b) the thin angular airfoils are made by bending a thin flat plate with a sharp ridge line and (c) the triangular airfoils have a flat lower surface. The thin angular airfoils and the triangular airfoils have three kinds of heights $h / c$ between $3 \%$ and $9 \%$ located at $30 \% c$ respectively. The height $h / c$ of airfoil is equivalent to the thickness $t / c$ for the triangular airfoil.

These models were made from aluminum and stainless steel sheets, and all of them have a smooth metal surface. When the sections of the sharp leading-edge and sharp trailing-edge are observed in detail, they are not perfectly sharp, but slightly thick, with a thickness ratio of $1 \% c$ as seen by cutting vertically. The wing models are rectangular in planform, with

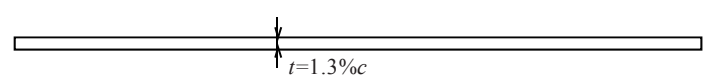

(a) Thin flat plate
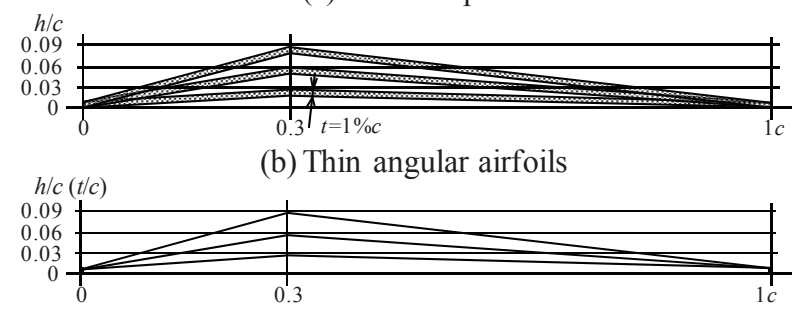

(c) Triangular airfoils

Fig. 2. Airfoils. 
an aspect ratio of 3 and a chord length of $40 \mathrm{~mm}$. These wing models were examined by changing the Reynolds number between 3,000 and 20,000 .

\section{Results and Discussion}

\subsection{The main aerodynamic characteristics of angular airfoils}

Figure 3 shows the main aerodynamic characteristics of all airfoils tested in this study; the maximum lift coefficient $C_{l \max }$, the minimum drag coefficient $C_{d \text { min }}$ and the maximum lift to drag ratio $(l / d)_{\max }$. A large $C_{l \max }$ is obtained by using the thin angular airfoil with larger height at $R e \geq 5,000$. As the Reynolds number increases to over 10,000 , a large $C_{l \max }$ following $9 \%$ and $6 \%$ thin airfoils is provided for the thick airfoil with the largest thickness of $9 \% c$. As the Reynolds number decreases to 3,000, the difference in $C_{l \max }$ according to the height ratio of the angular airfoils becomes small, and a larger $C_{l \max }$ is obtained for the $3 \%$ thin airfoil. $C_{l \max }$ of every angular airfoil was larger than that of the thin flat plate at any given Reynolds number.

$C_{d \text { min }}$ is larger than twice the theoretical laminar skin friction drag $2 C_{f}$ at every Reynolds number. It should be noted that $C_{d \min }$ of the $9 \%$ thin airfoil, which is largest at $R e \geq$ 10,000 , is small at $R e \leq 5000$.

The largest $(l / d)_{\max }$ is obtained for the thin angular airfoils with $6 \%$ height at $R e=20,000$. As the Reynolds number decreases, $(l / d)_{\max }$ of the $6 \%$ and $3 \%$ thin airfoils is the largest at $R e=10,000$, and it is largest for the smaller height of $3 \%$ at $R e=3,000$. At $R e \leq 5,000,(l / d)_{\max }$ of a thin flat plate was not low rather than that of the angular airfoil. The $(l / d)_{\max }$ of the thin airfoils was larger than that of the thick airfoils at $R e \leq$ 20,000 .

From these results, it was found that $C_{l \max }$ and $(l / d)_{\max }$ for the thin angular airfoils are larger than those for the triangular airfoils, and high $(l / d)_{\max }$ was obtained for the $6 \%$ airfoil at $R e$ $\geq 10,000$, and for the $3 \%$ airfoil at $R e \leq 5,000$.

The aerodynamic characteristics of each airfoil are discussed in the following sections.

\subsection{Flat plate}

Figure 4 shows the two-dimensional aerodynamic coefficients of the flat plate obtained by varying the Reynolds number between 3,000 and 20,000. The lift coefficient $C_{l}$ increases in proportion to the increase of angle of attack $\alpha$, and changes horizontally at $\alpha \geq 7 \mathrm{deg}$ at $R e=20,000$. The lift curves are close to the experimental $C_{l}$ measured by Mueller ${ }^{8)}$ at $R e=80,000$. Thus, the effect of the Reynolds number for a thin flat plate is comparatively small. However, the non-linearity of the lift curve was more remarkable as the Reynolds number decreased to 10,000 or less. The lift slope at $\alpha<3 \mathrm{deg}$ is a little smaller than that at a higher Reynolds number, and the lift slope at $\alpha>3 \mathrm{deg}$ is equivalent to the theoretical value for the two-dimensional lift slope of $2 \pi$. As the Reynolds number decreases to 5,000 or less, the maximum lift coefficient $C_{l \max }$ appears at $\alpha=10 \mathrm{deg}$ even for the thin flat plate. The slope of the pitching moment $C_{m, 0.25 c}$ at $25 \%$ chord is slightly positive at the small angle of attack range of $-2 \mathrm{deg} \leq \alpha \leq 2 \mathrm{deg}$. As the angle of attack increases, the slope
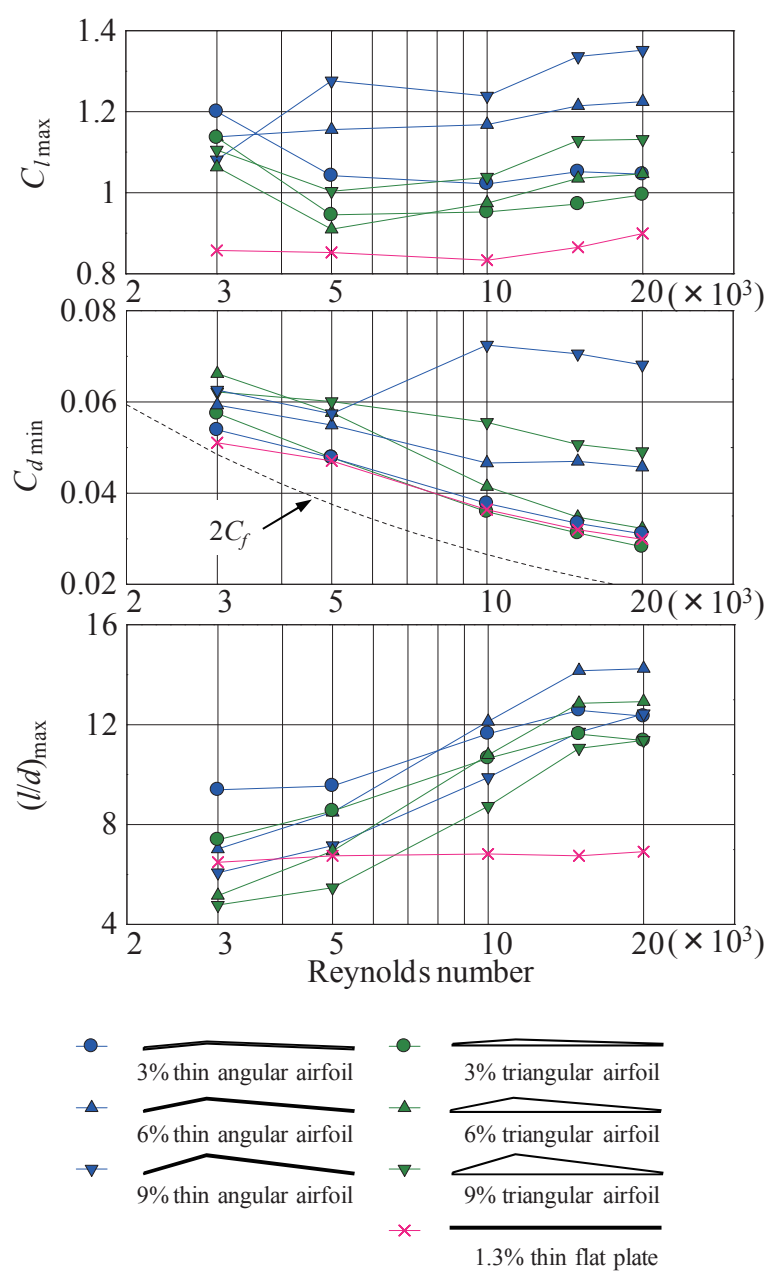

Fig. 3. Main aerodynamic characteristics of the airfoils.
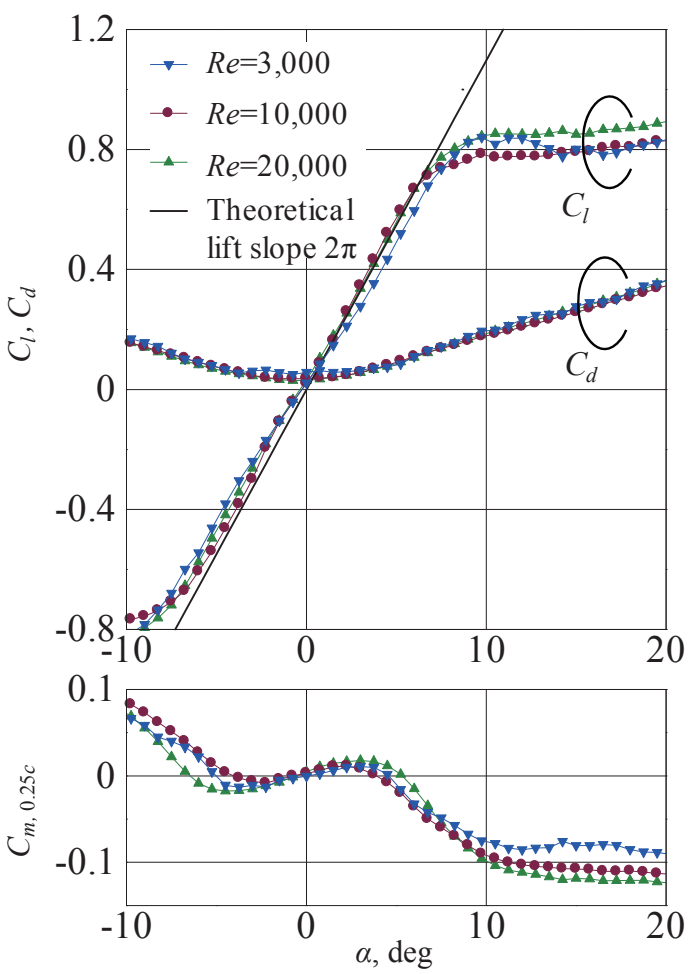

Fig. 4. Aerodynamic characteristics of the thin flat plate. 
of $C_{m, 0.25 c}$ changes gradually to negative with the increase of the lift slope. It is considered that the additional vortex lift by the leading-edge separation bubble causes the increase in the nose-up moment, but the moment changes to the nose-down direction with the increase in size of the separation bubble. The slight effect of Reynolds number is seen in the pitching moment coefficient $C_{m, 0.25 c}$, similar to the case for the lift curve; it changes to negative at a lower angle of attack as the Reynolds number decreases.

\section{3. $6 \%$ thin angular airfoil}

The aerodynamic characteristics of the thin angular airfoil with $6 \%$ height having the representative characteristics of angular shaped airfoils are discussed in this section.

Figure 5 shows the aerodynamic coefficients of the thin angular airfoil with the $6 \%$ height obtained by varying the Reynolds number between 3,000 and 20,000. Specifically, the aerodynamic characteristics of the airfoil at positive angles of attack were mainly discussed, because the differences in each Reynolds number on aerodynamic coefficients of the airfoil were small in the negative angles of attack range.

As seen in the results at $R e=10,000$, the non-linearity of the lift curve of the thin angular airfoil is more remarkable than that of the flat plate. The lift slope is small at $0 \mathrm{deg}<\alpha<$ $3 \mathrm{deg}$ and is large at $\alpha \geq 4 \mathrm{deg}$. It then gradually decreases and $C_{l}$ reaches the maximum as the angle of attack increases. As the angle of attack decreases to negative, the lift slope also gradually increases. The non-linearity lift slope seems to be caused by the separation vortex generated at the leading-edge. The flow of the upper surface of the wing will be laminar separation at a small angle of attack of $0 \mathrm{deg}<\alpha<3 \mathrm{deg}$. Then, the separation bubble appears at the leading-edge and gradually increases in size, as the angle of attack increases. When the separated flow of the leading-edge separation bubble reattaches at the back of an apex of its upper surface, the lift coefficient $C_{l}$ increases rapidly by the large negative pressure of the leading-edge vortex. As the angle of attack decreases to negative, the separation bubble appears at the lower surface of the airfoil near the leading-edge and lift slope increases slightly due to the negative vortex lift.

The drag coefficient $C_{d}$ increases with the increase of $C_{l}$, but the ratio of the increase in $C_{d}$ decreases as $C_{l}$ increases rapidly. At that time, the maximum lift to drag ratio $(l / d)_{\max }$ is provided.

The pitching moment coefficient $C_{m, 0.25 c}$ show a negative value at the zero-lift angle due to the effect of positive camber, and $C_{m, 0.25 \mathrm{c}}$ increases as $C_{l}$ increases largely due to the separation bubble. At that time, the separation region near the trailing-edge decreases by the leading-edge separation bubble, and the large negative pressure generated near the leading-edge acts on the airfoil in the nose-up direction. As discussed later, these phenomena are pronounced in the pressure distribution of the airfoil.

As the Reynolds number decreases to 5,000 or less, the non-linear lift slope is more remarkable, and the lift slope of $\alpha$ $>2$ deg decreases, but increases rapidly at $\alpha=7 \mathrm{deg}$ for $R e=$ 5,000 and $\alpha=10 \mathrm{deg}$ for $R e=3,000$ respectively. $C_{l \text { max }}$ becomes as large as that at $R e \geq 10,000$. In contrast, the change in $C_{d}$ due to the leading-edge separation bubble is smaller than that at $R e=10,000$. At the same time, the change in $C_{m, 0.25 c}$ is also small.

As the Reynolds number increases to 20,000 , the nonlinearity of the lift slope is small in the lift curve. This
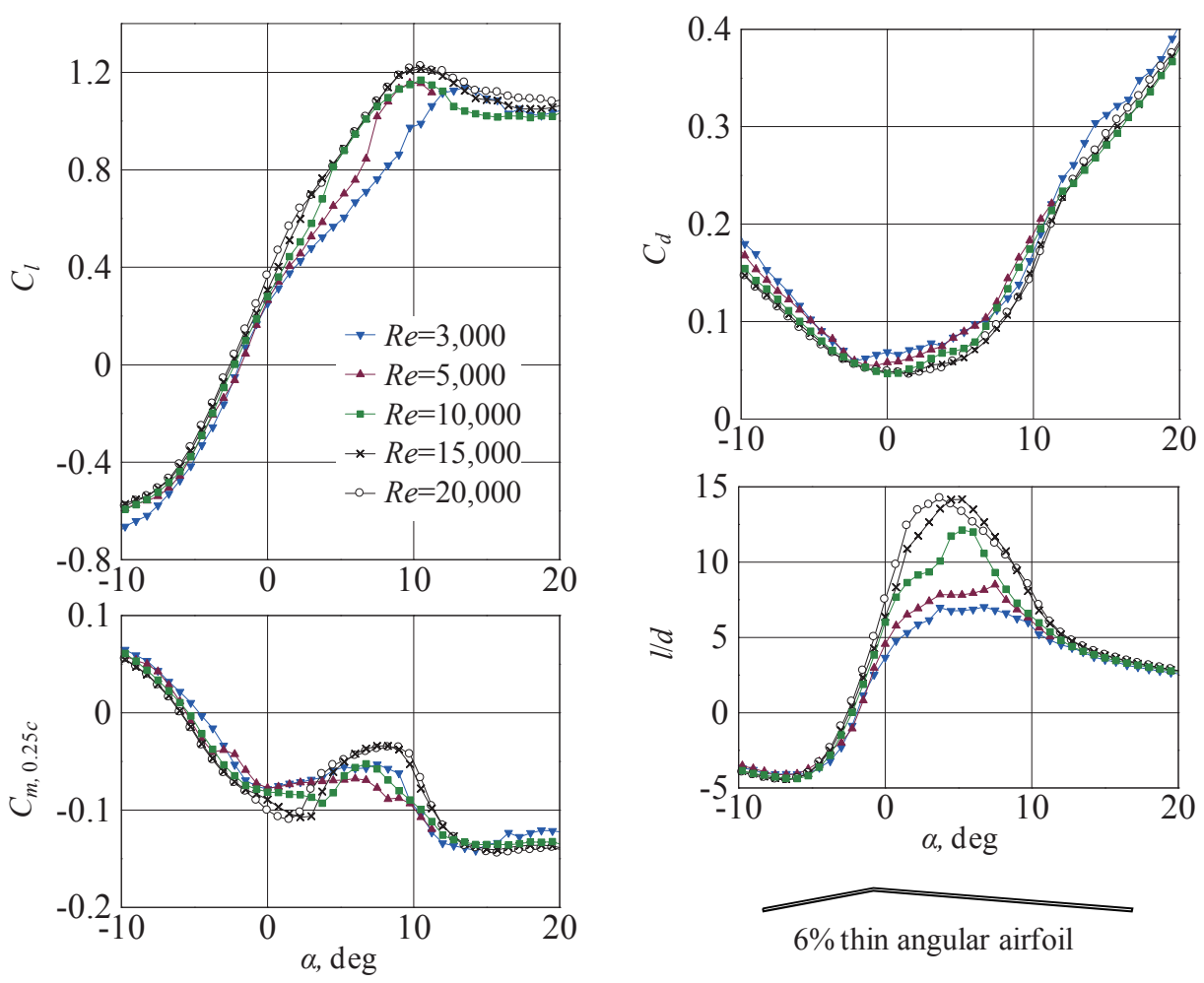

Fig. 5. Aerodynamic characteristics of the $6 \%$ thin angular airfoil. 
phenomenon was also seen by integrating the pressure distributions of the airfoil. Although $C_{d}$ increases continuously as the angle of attack increases, the increase rate of $C_{d}$ increases largely at $\alpha>5 \mathrm{deg}$. The negative value of $C_{m, 0.25 c}$ at the zero-lift angle is slightly larger than that at $R e=10,000$, and the negative $C_{m, 0.25 c}$ decreases remarkably at $\alpha \geq 4 \mathrm{deg}$. It was confirmed that the change in lift is small, whereas the change in drag and pitching moment increase due to the leading-edge separation bubble at a large Reynolds number.

As seen in the curves of the lift-to-drag ratio $l / d$ in Fig. 5, $(l / d)_{\max }$ is obtained near the angle of attack where the leading-edge separation bubble generated, and $(\mathrm{l} / \mathrm{d})_{\max }$ is over 14 at $\operatorname{Re}=20,000$ and 6 at $\operatorname{Re}=3,000$.

\subsection{Pressure distribution of $6 \%$ thin angular airfoil}

Figures 6(a)-(c) show the pressure coefficient $C_{p}$ distributions of the upper and lower surfaces of the $6 \%$ thin angular airfoil with various angles of attack as the Reynolds numbers varied from 3,000 to 20,000 . From these results, as the angle of attack increases, the pressure coefficient $C_{p}$ of the upper surface decreases and that of the lower surface increases.

Figure 6(a) shows $C_{p}$ distributions at $R e=3,000$. At $\alpha=-3$ deg and $0 \mathrm{deg}$, positive $C_{p}$ of the upper surface near the leading-edge decreases to an apex of an angular surface, and increases toward the trailing-edge. A large negative $C_{p}$ of the lower surface increases at the rear of the airfoil. That is, the leading-edge separation bubble seems to be due to appear at the lower surface. As the angle of attack increases, $C_{p}$ of the upper surface near the leading-edge decreases due to the large wind velocity over the sharp leading-edge, and that of the lower surface shows a positive value for every position of the chord. At $\alpha=6 \mathrm{deg}, C_{p}$ at leading-edge is very large and increases to the trailing-edge continuously. It seems to be caused by the leading-edge separation bubble generated on the leading-edge of the wing. However, the distribution of $C_{p}$ is not that of the distinctive separation bubble. The lift coefficient $C_{l}$ increases rapidly, as the bubble becomes larger slightly at $\alpha=9 \mathrm{deg}$. When $C_{p}$ at the leading-edge becomes large at $\alpha=12 \mathrm{deg}$, the decrease of $C_{p}$ toward the trailing-edge is small and the flow separation extends largely.

As the Reynolds number increases, the similar distributions of $C_{p}$ are seen in Fig. 6(b). However, $C_{p}$ near the trailing-edge at $R e=10,000$ is slightly larger than that of a smaller Reynolds number. At $\alpha=3 \mathrm{deg}, C_{p}$ of the upper surface near the leading-edge increases once at the back of the leading-edge, but decreases at the apex of an angular surface. Although the leading-edge separation bubble is generated, the lift coefficient $C_{l}$ is still low because the location of the reattachment is in front of an apex. As the angle of attack increases to $6 \mathrm{deg}$, a large negative $C_{p}$ of the leading-edge increases rapidly at the $60 \%$ of the chord, and $C_{p}$ near the trailing-edge becomes smaller than that of the smaller angle of attack, and $C_{l}$ is remarkably large. When $C_{p}$ of the upper surface near the leading-edge is small at $\alpha=12 \mathrm{deg}$, a flow separation extends over the airfoil and $C_{l}$ decreases.

Distinctive distributions of $C_{p}$ are seen at $R e=20,000$, as shown in Fig 6(c). As $C_{p}$ at the leading-edge decreases at the angle of attack $\alpha \geq 6 \mathrm{deg}$, a horizontal slope of $C_{p}$ is seen near

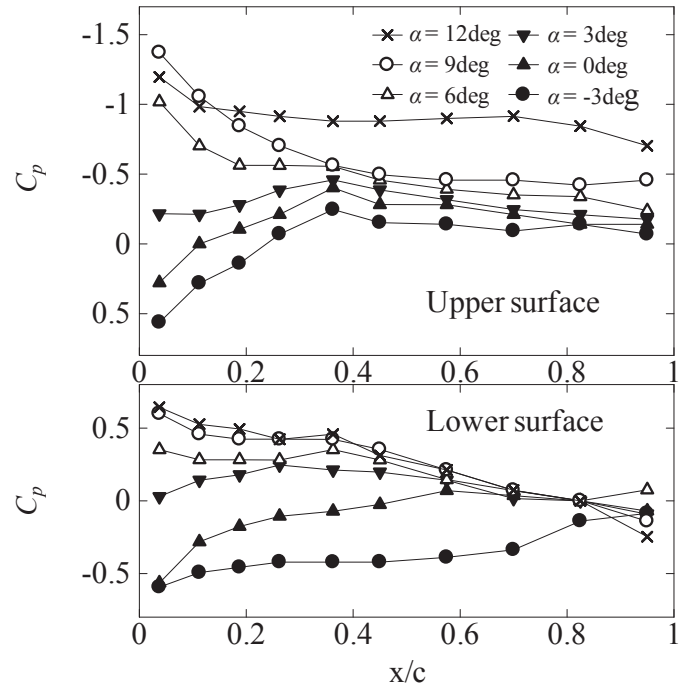

(a) $\operatorname{Re}=3,000$

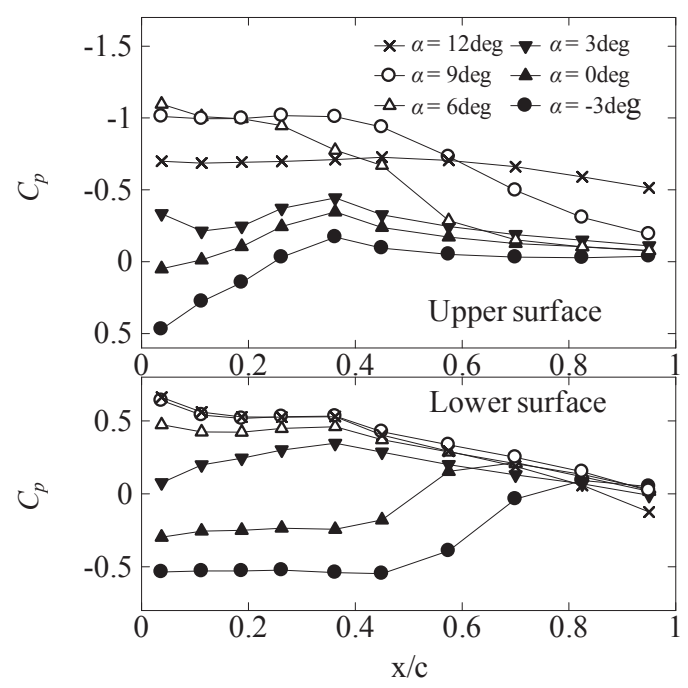

(b) $R e=10,000$

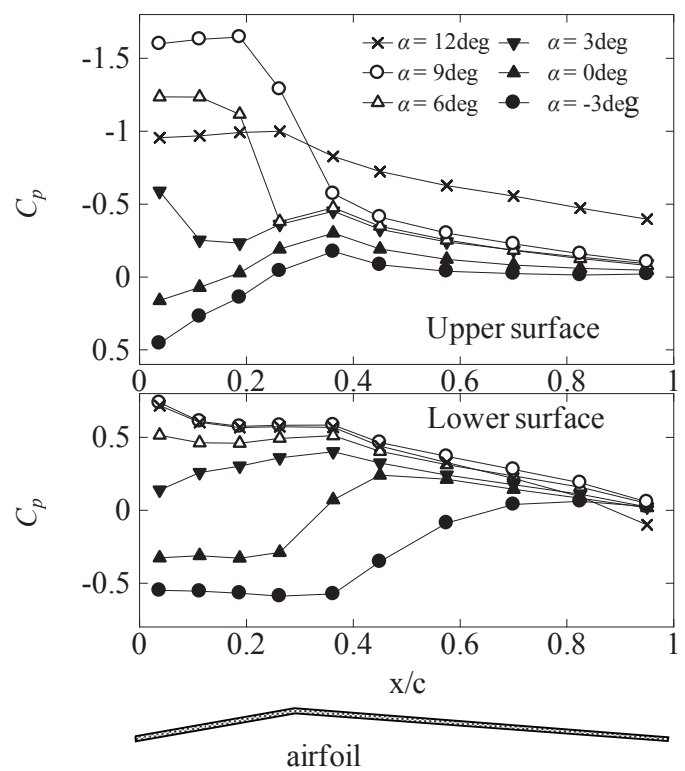

(c) $R e=20,000$

Fig. 6. Pressure distributions of the $6 \%$ thin angular airfoil. 
the leading-edge and $C_{p}$ increases rapidly toward the trailing-edge. This distribution of $C_{p}$ shows the leading-edge separation bubble. That is, the transition from laminar flow to turbulent flow occurs in the flow separated at the sharp leading-edge, and the flow reattaches near the trailing-edge. At that time, the negative $C_{m, 0.25 c}$ decreases because the large $C_{p}$ is in front of an apex.

As the angle of attack decreases to the negative value, the distribution of $C_{p}$ of the lower surface shows the leading-edge separation bubble similar to the case for the upper surface, and the position of the reattachment of the flow moves to the trailing-edge according to the decrease of angle of attack.

\subsection{Thickness effect of triangular airfoil}

Figure 7(a) shows the results obtained by changing the thickness between $3 \% c$ and $9 \% c$ at $R e=3,000$. The lift slopes of every airfoil at the small angle of attack range of $\alpha>0 \mathrm{deg}$ are smaller than the theoretical $2 \pi$. The lift slope increases suddenly at $\alpha=12 \mathrm{deg}$ for the $3 \%$ airfoil, at $\alpha=10 \mathrm{deg}$ for the $6 \%$ airfoil and at $\alpha=7 \mathrm{deg}$ for the $9 \%$ airfoil. The maximum lift coefficient $C_{l \text { max }}$ shows the similar value regardless of the thickness. The angle of attack of $C_{l \text { max }}$ is smallest for the $3 \%$ airfoil and largest for the $9 \%$ airfoil. The drag coefficient $C_{d}$ shows almost the same value for all airfoils at the zero lift angle. The pitching moment $C_{m, 0.25 c}$ at the $25 \% \mathrm{c}$ shows a similar value for all airfoils, similar to the case for $C_{l}$ at the zero lift angle. The negative value of $C_{m, 0.25 c}$ increases according to the rapid increase of the lift slope, and then it shows approximately -0.1 at $\alpha=20$ deg regardless the airfoil thickness. As seen in the polar curve, the maximum lift-to-drag ratio $(l / d)_{\max }$ for the $3 \%$ thick airfoil is largest of all at $R e=3,000$.

Figure 7(b) shows the results obtained by changing the maximum thickness ratio at $R e=10,000$. The non-linearity of the lift slopes is small except that of the $9 \%$ thick airfoil. The lift slope of the $9 \%$ thick airfoil increases dramatically at $\alpha=8$ deg, and $C_{l \max }$ shows the largest of all airfoils. $C_{l}$ for the $9 \%$ thick airfoil decreases at $\alpha=12 \mathrm{deg}$. $C_{m, 0.25 c}$ of the $9 \%$ thick airfoil shows the large negative value around $\alpha=0 \mathrm{deg}$, and the non-linear shape of $C_{m, 0.25 c}$ curve of the $6 \%$ thick airfoil was more remarkable in comparison with that of $R e<5,000$.

When the Reynolds number increases to 20,000 as shown in Fig. 7(c), the non-linearity of the lift slope is small in comparison with the case of $R e=10,000$. In particular, $C_{l}$ for the $3 \%$ airfoil shows the linear lift slope. However, $C_{d}$ and $C_{m, 0.25 c}$ vary as the angle of attack increases. $C_{m, 0.25 c}$ for all airfoils increases close to zero at $\alpha=6 \mathrm{deg}$ for the 3\% airfoil, $\alpha=8 \mathrm{deg}$ for the $6 \%$ airfoil and $\alpha=11 \mathrm{deg}$ for the $9 \%$ airfoil.

From these results, it is considered that the airfoil thickness affects the non-linearity of the lift slope, similar to the case for the thin angular airfoil. As the thickness increases, the lift slope decreases at $R e=3,000$, and the change in lift slope is small at $R e=20,000$. The increase of $C_{l \max }$ in proportion to the increase of thickness was not seen at $R e=3,000$.

\subsection{Comparison of thin airfoil and thick airfoil}

Figure 8 shows a comparison of aerodynamic characteristics between the thin angular airfoil and the triangular airfoil with $6 \%$ height at $R e=10,000$. In addition, the theoretical $C_{l}$ and $C_{m, 0.25 c}$ obtained by the potential analysis for thin angular
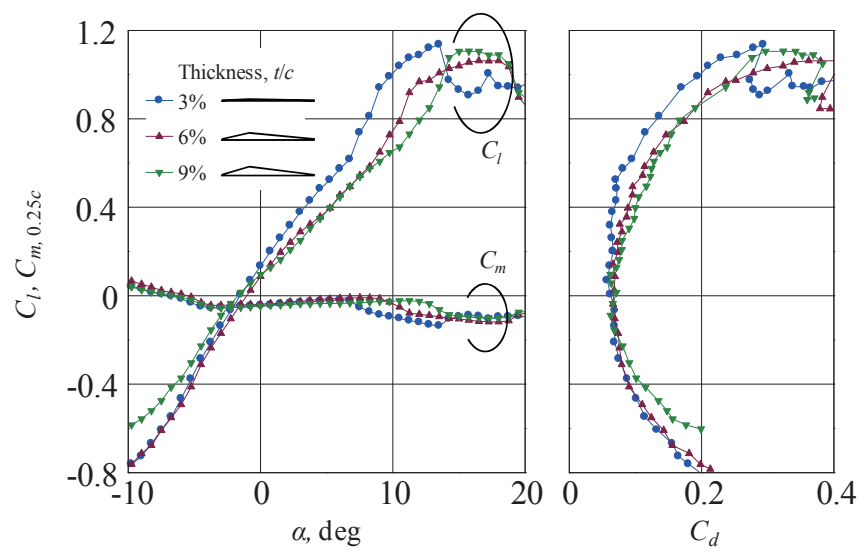

(a) $R e=3,000$
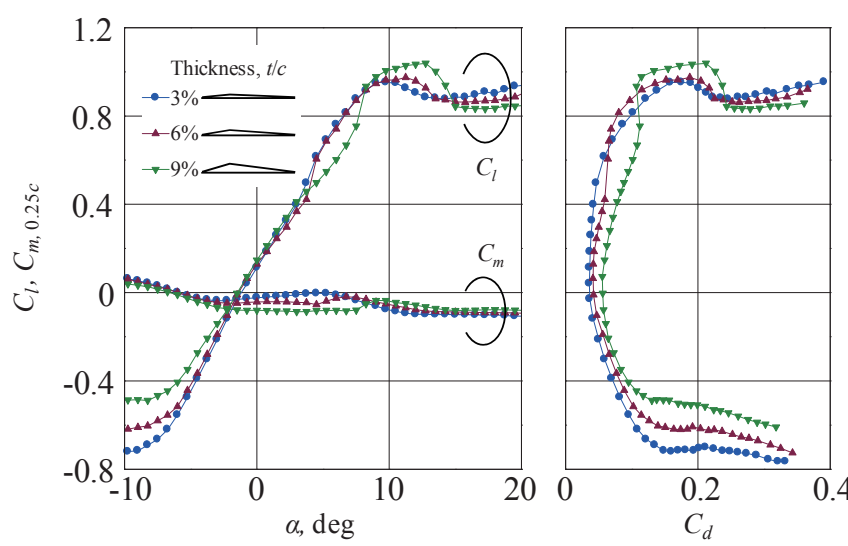

(b) $R e=10,000$
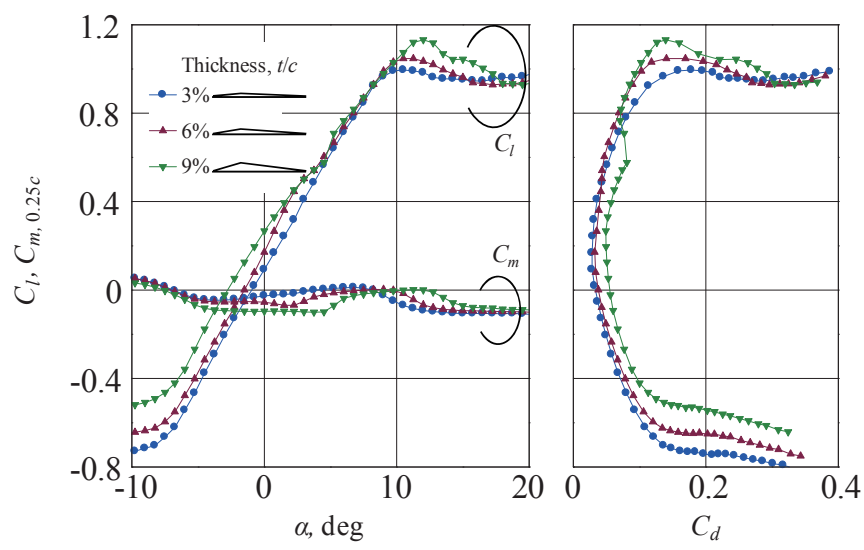

(c) $R e=20,000$

Fig. 7. Thickness effects of the triangular airfoils.

airfoil ${ }^{9)}$ were also represented in the figure. The difference in the theoretical zero-lift angles is due to the camber of the airfoils, and $C_{l}$ of the airfoil with larger camber is large at the same angle of attack. However, the experimental $C_{l}$ is not proportional to the angle of attack at low Reynolds number, whereas the theoretical lift slope is constant of $2 \pi$. The differences in experimental $C_{l}$ for the airfoils with different cambers are very small in comparison with the differences in the theoretical $C_{l}$ at low or negative angle of attack. However, when the separation bubble appeared at $\alpha \geq 4 \mathrm{deg}$, those experimental differences are close to the theoretical values. 


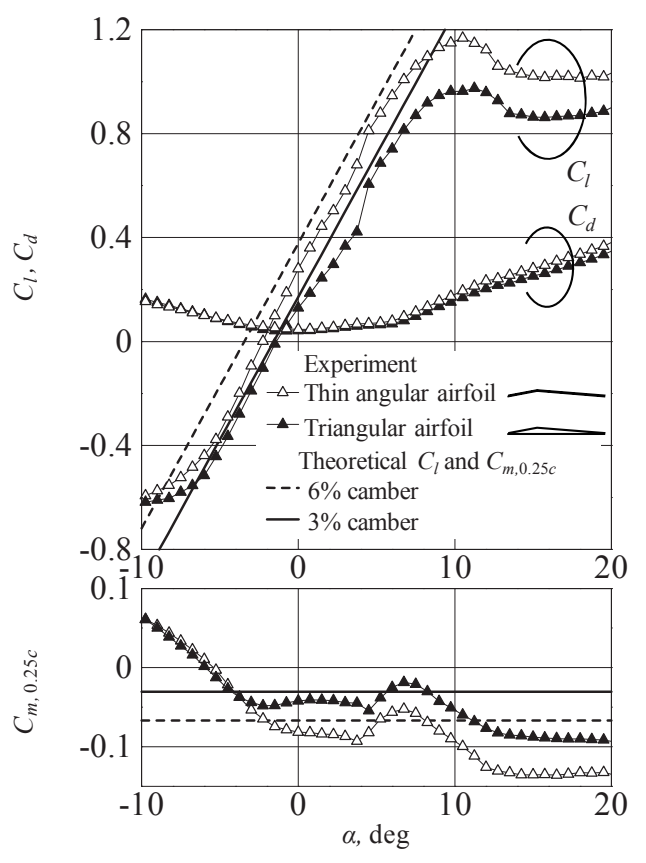

Fig. 8. Comparison between the thin angular airfoil and the triangular airfoil with $6 \%$ heights at $R e=10,000$.

Further, as seen in the lift slope in Fig. 8, it is recognized that the rapid increase in $C_{l}$ due to the leading-edge separation bubble occurs at the same angle of attack for both thin and thick airfoils. This phenomenon shows that the non-linearity lift curve generated by the leading-edge separation bubble depends on the shape of the upper surface of the airfoil. On the other hand, the magnitude of $C_{l}$ depends on the shape of the lower surface.

As stated above, generally, the maximum lift coefficient $C_{l \text { max }}$ and the maximum lift to drag ratio $(l / d)_{\max }$ for the thin airfoil are larger than those for the thick airfoil at the present low Reynolds number. Because, the differences in $C_{l}$ between thin airfoil and thick airfoil are large, whereas the differences in $C_{d}$ are comparatively small.

The pitching moment coefficient at the zero lift angle, that is $C_{m, C l=0}$, is similar to the theoretical $C_{m, 0.25 c}$, although the experimental $C_{m, 0.25 c}$ shows large negative value with the increase of the angle of attack. It seems to be caused by a nose-up moment due to a laminar separation. This negative moment approaches to the theoretical value, as the separation bubble is generated.

\section{Conclusions}

The following results were obtained by the wind tunnel tests of angular-shaped airfoils at low Reynolds numbers between 3,000 and 20,000:

(1) The maximum lift coefficient $C_{l \max }$ and the maximum lift to drag ratio $(l / d)_{\max }$ for the thin airfoils are larger than those of thick airfoils, and are large even for the airfoils composed of a line pattern at the present low Reynolds number.

(2) The aerodynamic characteristics of the triangular airfoils are affected in the airfoil thickness by changing the Reynolds number. High $(l / d)_{\max }$ was obtained for the $6 \%$ airfoil at $R e \geq$ 10,000 , and for the 3\% airfoil at $R e \leq 5,000$.

(3) The lift curves are characterized by the existence of the leading-edge separation bubble. The non-linearity of the lift curve is small at $R e=20,000$, and it becomes large as the Reynolds number decreases.

(4) The non-linear lift curve depends on the shape of the upper surface of the airfoil and the magnitude of $C_{l}$ depends on the shape of its lower surface.

(5) The pressure coefficients $C_{p}$, specifically near the leading-edge, show the distinctive distribution by the Reynolds number.

(6) In particular, the large negative pressure of $C_{p}$ near the leading-edge make the negative $C_{m, 0.25 c}$ decrease remarkably as the Reynolds number increases. Therefore, the non-linearity of the pitching moment curve is more remarkable as the Reynolds number increases.

\section{References}

1) Asai K. and Nagai H., "Planetary Locomotion-New Options for Exploration on Planets," Aeronaut. Space Sci. Japan, 60, 11 (2012), pp. 421-426. (in Japanese)

2) Okamoto, M., Yasuda, K. and Azuma. A., "Aerodynamic Characteristics of the Wings and Body of a Dragonfly," J. Exp. Biol., 199 (1996), pp. 281-294.

3) Azuma, A., Okamoto, M. and Yasuda, K., "Aerodynamic Characteristics of Wing at Low Reynolds Number," Fixed and Flapping Wing Aerodynamics for Micro Air Vehicle Applications, edited by T. J. Mueller, Progress in Astronautics and Aeronautics, 195, AIAA, Reston, VA (2001), pp. 341-398.

4) Kesel, A. B., "Aerodynamic Characteristics of Dragonfly Wing Sections Compared With Technical Aerofoils," J. Exp. Biol., 203 (2000), pp. 2125-2135.

5) Sunada, S., Yasuda, T., Yasuda, K. and Kawachi, K., "Comparison of Wing Characteristics at an Ultralow Reynolds Number," J. Aircraft, 39 (2002), pp. 331-338.

6) Okamoto, M. and Azuma, A., "Aerodynamic Characteristics at Low Reynolds Numbers for Wings of Various Planforms," AIAA J., 49, 6, (2011), pp.1135-1150.

7) Crompton, M. J. and Barrett, R.V., "Investigation of the Separation Bubble Formed Behind the Sharp Leading-edge of a Flat Plate at Incidence," Proceedings of the Institution of Mechanical Engineers, 214 (2000), pp. 157-176.

8) Torres, G. E. and Mueller, T. J., "Aerodynamic Characteristics of Low Aspect Ratio Wings at Low Reynolds Numbers," Fixed and Flapping Wing Aerodynamics for Micro Air Vehicle Applications, edited by T. J. Mueller, Progress in Astronautics and Aeronautics, 195, AIAA, Reston, VA, (2001), pp. 115-141.

9) Azuma, A., Okamoto, M., "Theoretical study on two-dimensional aerodynamic characteristics of unsteady wings," J. Theor. Biol., 234 (2005), pp.67-78 